\title{
Interpretation of Certain Features of the Interface Stress vs. Electrode Potential Curves of Gold with the Help of Dual Dynamic Voltammetry
}

\author{
G.G. Láng ${ }^{\mathrm{a}}$, N. Kovács ${ }^{\mathrm{a}}$, Z. Dankházi ${ }^{\mathrm{b}}$, S. Vesztergoma ${ }^{\mathrm{a}}$, and M. Ujvária ${ }^{\mathrm{a}}$ \\ a Institute of Chemistry, Department of Physical Chemistry\&Laboratory of \\ Electrochemistry and Electroanalytical Chemistry, Eötvös Loránd University, \\ Pázmány P. s. 1/A, H-1117 Budapest, Hungary \\ ${ }^{b}$ Institute of Physics, Department of Material Physics, Eötvös Loránd University, \\ Pázmány P. s. 1/A, H-1117 Budapest, Hungary
}

It is a widespread opinion that the electrochemical behavior of gold in aqueous media has been thoroughly studied, and the interface stress changes of gold can be explained by monolayer oxide formation/removal and adsorption phenomena. However, in spite of the voluminous literature on this subject, an understanding of these systems and processes is far from complete. The combination of in-situ interface stress measurements and dual dynamic voltammetry brings new insights into the complexity of such systems. One remarkable result is that in the oxide reduction region of gold in sulfuric acid solutions a sudden change in the slope of the interface stress vs. electrode potential curve recorded during the negative-going scan of the cyclic voltammogram can be observed. By applying the dual dynamic voltammetric method to a gold/gold rotating ring-disk electrode (RRDE) it could be shown that the sharp change in the slope of the surface stress vs. potential curve recorded in the oxide reduction region of gold in sulfuric acid solutions is accompanied by the formation of soluble, electrochemically reducible gold species which can be detected at the ring electrode.

\section{Introduction}

When molecules and/or ions are adsorbed on a solid surface (solid/liquid interface), the adsorbed species affect the energetics of the interface and the electrical properties such as the conductance, resistance and capacitance. The formation of an interface between an electronic conductor and an ionic conductor is accompanied by a spatial separation of charge, such that electric potential differences may appear between the adjacent phases. The thermodynamic properties of this region (e.g. the interface between a metal and an electrolyte solution) directly influence the electrochemical processes, therefore, an understanding of the thermodynamics of interfaces is of importance to all surface scientists and electrochemists. The intensive parameter conjugate to surface area (1) (often called the "surface tension", "specific surface energy", "interfacial tension", "interface stress" or "surface stress") is an important parameter in the thermodynamic theory of electrochemical systems, since from a thermodynamic point of view electrodes are in fact capillary systems, because any interaction between the electronic conductor and the remainder of the system takes place via the interface region (2). It is not 
surprising, therefore, that the interest in the structure and properties of charged solid|liquid interfaces has increased steadily throughout the past decades and stimulated interesting discussions and debates in the electrochemical community $(3,4)$. Nevertheless, the understanding of fundamental aspects of interfacial electrochemistry is also important for other fields, such as materials science, nanoscience, biology, medical science, sensor and battery research, as well as for technologies based on electrochemical techniques.

During the past decades several methods were suggested for measurements of changes of the "surface tension" ("interface stress") of "solid electrodes". (A solid electrode is a multicomponent, multiphase electrochemical system that contains an electronically conducting solid phase in contact with an ionically conducting liquid phase). The suggested methods usually yield changes in surface stress as a function of the electrode potential (5). In most cases the variation in interface stress is obtained indirectly by measuring the potential dependence of the strain (i.e. electrode deformation), and the change of the stress is calculated using the appropriate form of Hooke's law. The experimental data are cited in several papers as "proofs" for different theories and statements, but often without any critical analysis of the experimental methods, or the reliability of the experimental data. Gold in contact with different electrolyte solutions is a particularly attractive model system for such investigations (3).

It is a widespread opinion that the electrochemical behavior of gold in aqueous media has been thoroughly studied, and the interface stress changes of gold can be explained by monolayer oxide formation/removal and adsorption phenomena. The oxide formation on gold has been the subject of numerous electrochemical studies involving single- or polycrystalline gold surfaces in contact with different electrolyte solutions (6-9). On the basis of these studies the coexistence of (at least) three different compounds of gold at the surface was assumed and a mechanism for the early stages of oxidation of gold based on an increasing degree of adsorption of $\mathrm{OH}$ groups at the surface was proposed accounting for the competition of anion adsorption as well. According to the results the strength of the metal-oxygen bond differs for the different species which are formed ranging from a low value, approaching the condition of physical O-adsorption, to a relatively high bond energy corresponding to chemical surface oxide.

Nevertheless, there are some peculiarities that are worth being discussed and clarified:

i) On the basis of rotating ring disk electrode (RRDE) measurements an additional dissolution process besides the dissolution of gold during oxide formation that is corresponding to the oxide reduction was proposed long time ago by Cadle and Bruckenstein (10). More recently, utilizing the rotating ring-disk electrode (RRDE) setup and the phase-shifted dual cyclic voltammetry method it could be shown that the reduction of the surface oxide layer of a polycrystalline gold disk immersed into sulfuric acid solutions is accompanied by the formation of soluble, electrochemically reducible gold species which can be detected on the ring electrode (11). This observation has been confirmed by other studies such as (12) and (13-16).

ii) In some cases a sudden change in the slope of the interface stress vs. electrode potential curve recorded during the negative-going scan of the cyclic voltammogram could be observed in the oxide reduction region of gold in perchloric and sulfuric acid solutions $(17,18)$.

Unfortunately, the results reported in the literature are comparable to a limited extent only because they were obtained under different experimental conditions: in different solutions, at different sweep rates, at different temperatures and concentrations, etc. The 
aim of this study is to show that under well-controlled experimental conditions the formation of a soluble gold species and the sudden change in the slope of the interface stress vs. electrode potential curve occur within the same potential range suggesting a close link between the two phenomena.

\section{Experimental}

\section{Dual Dynamic Voltammetry}

"PINE AFE7R8" RRDE tips (collection efficiency: 22\%) were used in the dual dynamic voltammetry experiments in a standard four electrode cell; both the disk (geometric surface area $A_{\mathrm{d}}=0.1642 \mathrm{~cm}^{2}$,) and the ring $\left(A_{\mathrm{r}}=0.0370 \mathrm{~cm}^{2}\right.$ ) were made of polycrystalline gold. The gap size between the ring and the disk was $178 \mu \mathrm{m}$. The electrode surface was firmly polished with diamond suspensions (Struers) down to a diamond particle size of $0.25 \mu \mathrm{m}$. The RRDE tip was then rinsed with pure ethanol and Milli-Q water, after that it was cleaned ultrasonically for one minute, and washed extensively with ultrapure water. All the glass parts used in the experiments were immersed in piranha solution for several hours, rinsed with de-ionized water, and cleaned by steam.

The electrochemical cell contained a separate reference compartment being connected to the cell by a Luggin capillary positioned $\sim 1 \mathrm{~mm}$ below the RRDE surface. $\mathrm{A} \mathrm{NaCl}$ saturated calomel electrode (SSCE) was applied as reference and a gold plate with large surface was used as a counter electrode. The $0.05 \mathrm{~mol} \cdot \mathrm{dm}^{-3} \mathrm{H}_{2} \mathrm{SO}_{4}$ solution was made by dilution of concentrated sulfuric acid (Merck, "for analysis" or "ultrapure") with high purity ("Milli-Q") water. Measurements were carried out at room temperature and in Ar atmosphere. Solutions were purged with oxygen-free argon (Linde 5.0) before use and an inert gas blanket was maintained throughout the experiments.

The RRDE measurements were carried out at $25.0^{\circ} \mathrm{C}$ by using our self-built electrochemical workstation (19) incorporating a bi-potentiostatic assembly of XPot devices manufactured and modified specially for our needs by Zahner Elektrik, Kronach, Germany. The oxide on the gold surface was formed by potential cycling up to the so called "Burshtein minimum" at which electrode is assumed to be covered with one monolayer of adsorbed oxygen and the formation of three-dimensional structures ("higher Au-oxides") is negligible. The bending beam experiments were performed within the same potential range.

\section{Bending beam experiments}

The changes of the interface stress $\left(\Delta \gamma_{\mathrm{s}}\right)$ of an electrode consisting of a thin metal film on one side of an insulating plate in contact with an electrolyte solution can be estimated from the changes of the radius of curvature $\left(R_{\mathrm{c}}\right)$ of the cantilever by using an expression based on Stoney's equation $(20,21)$ :

$$
\Delta \gamma_{\mathrm{s}}=\frac{E_{\mathrm{s}} t_{\mathrm{s}}^{2}}{6\left(1-v_{\mathrm{s}}\right)} \cdot \Delta\left(1 / R_{\mathrm{c}}\right)=k_{\mathrm{i}} \Delta\left(1 / R_{\mathrm{c}}\right) \approx k_{\mathrm{i}} \frac{\Delta y}{2 n_{\mathrm{s}, \mathrm{i}} h w}
$$


where $E_{\mathrm{s}}, v_{\mathrm{s}}$ and $t_{\mathrm{s}}$ are Young's modulus, Poisson's ratio, and thickness of the plate, respectively, $h$ is the distance between the lower end of the electrode holder and the reflection point of the laser beam; $l_{1}$ is the distance between the optical window and the reflection point on the electrode, $l_{2}$ is the distance between the optical window and the position sensitive (photo)detector (PSD) plane, $w=l_{1}+l_{2}$, and $\Delta y$ is the change of the position of the light spot on the PSD (see Fig.1), and $n_{\mathrm{s}, \mathrm{i}}$ is the refractive index of the solution (22,23). The values of $\Delta\left(1 / R_{\mathrm{c}}\right)=\Delta \gamma_{\mathrm{s}} / k_{\mathrm{i}}$ can be calculated, if the changes of the deflection angle of a laser beam mirrored by the plate are measured $(3,5)$.

Cantilever beams used for the measurement of interface stress changes were made by evaporating a $100 \mathrm{~nm}$ thick gold layer on a very thin layer of titanium evaporated on one side of a glass plate (supplier: Matsunami Glass Ind., Ltd., Japan, total length: $l_{\mathrm{s}}=60.0 \mathrm{~mm}$, width: $w_{\mathrm{s}}=5.0 \mathrm{~mm}$, thickness: $t_{\mathrm{s}}=147 \mu \mathrm{m}$, Poisson's ratio: $v_{\mathrm{s}}=0.230$, Young's modulus: $E_{\mathrm{s}}=7.09 \cdot 10^{10} \mathrm{~N} \mathrm{~m}^{-2}$, refractive index: $n_{\mathrm{g}}=1.522$ ) after a careful cleaning of the surface of the substrate. X-ray diffraction patterns revealed that the $\mathrm{Au}$ films were preferentially (111)-oriented. The geometric area of the electrode was $A=4.0 \mathrm{~cm} \times 0.50 \mathrm{~cm}=2.0 \mathrm{~cm}^{2}$. According to Eq. [1], $k_{\mathrm{i}}=331.6 \mathrm{~N}$.

A battery-powered potentiostat (Jaissle Model 1001 T-NC) was used for the interface stress measurements. Control voltage was supplied by a function generator (HewlettPackard 3314A) or by a data acquisition card. A National Instruments 6034E DAC was used for the computer-based acquisition of the analog output signals of the PSD and the potentiostat. The software for controlling and data acquisition was developed in the National Instruments LabVIEW ${ }^{\circledR}$ environment. A position-sensitive detector (Hamamatsu Inc., S1300) attached to a signal processing unit (Hamamatsu C4757), the He-Ne laser (Melles Griot 05-LHP-151) and the electrochemical cell (gas-tight cell made of Pyrex ${ }^{\circledR}$ glass) were assembled on an optical bench in order to avoid vibrations. The distance between the level of the solution in the cell and the reflection point of the laser beam $(h)$ was measured with the help of a cathetometer (type KM-6).

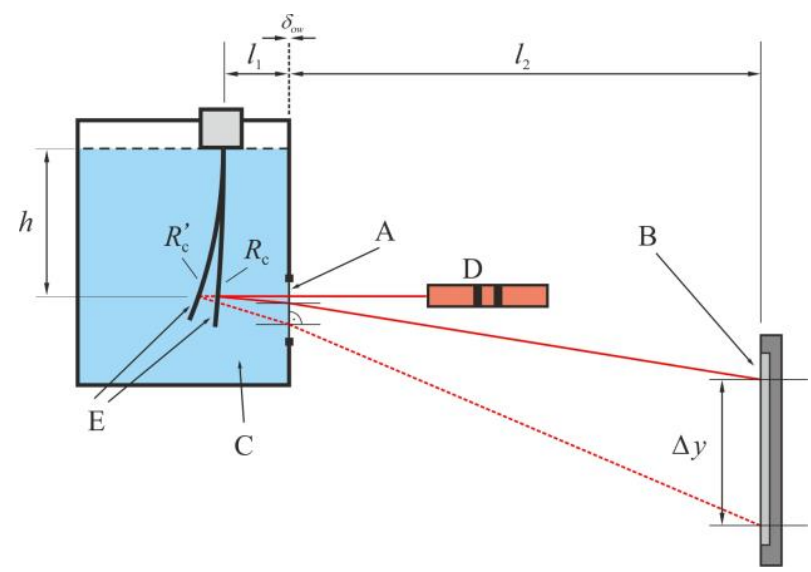

Figure 1. Schematic representation of a typical electrochemical (optical) bending beam setup (normal incidence of the light beam). $\Delta y$ is the displacement of the light spot on the position sensitive detector (PSD) if the radius of curvature changes from $R_{\mathrm{c}}$ to $R_{\mathrm{c}}{ }^{\prime}, l_{1}$ is the distance between the clamped end of the probe (cantilever, electrode) and the optical window, $l_{2}$ is the distance between the optical window and the photodetector (PSD), $h$ is the distance between the solution level and the reflection point, $\delta_{\text {ow }}$ is the thickness of the optical window, respectively. A: optical window, B: position sensitive photodetector (PSD), C: electrolyte solution, D: laser, E: electrode (cantilever) in different positions. 
The electrochemical bending beam experiment was performed in a gas-tight cell made of Pyrex glass equipped with an optical window (made of optical crown glass) at $25.0^{\circ} \mathrm{C}$. The electrochemical cell and the sulfuric acid solution (aliquots of the same solution prepared for the dual dynamic voltammetry measurements) have been purged with high purity, oxygen-free argon gas (Linde 5.0) before use and an argon gas blanket was maintained throughout the experiments. A conventional three-electrode configuration was used, with a cantilever working electrode, a counter electrode (a cylindrical gold plate in contact with the electrolyte solution), and an aqueous sodium chloride saturated calomel reference electrode (SSCE). The refractive index of the $0.05 \mathrm{~mol} \cdot \mathrm{dm}^{-3} \mathrm{H}_{2} \mathrm{SO}_{4}$ solution was measured at $25.00{ }^{\circ} \mathrm{C}$ with a Zeiss PR 2 refractometer $\left(n_{\mathrm{s}, \mathrm{i}}(\lambda=633 \mathrm{~nm})=1.3320\right)$.

\section{Results and discussion}

In conventional RRDE experiments the potential (or the current) of at least one of the working electrodes is held constant. It was recently shown, however, that independent and dynamic potential programs can also be applied simultaneously to the disk and the ring electrodes (11,24-26). By means of "dual potentiodynamic control", the sensitivity of the RRDE system can significantly be increased. As an example of dual dynamic potential control, dual cyclic voltammetry was applied to investigate the dissolution of gold upon potential cycling.

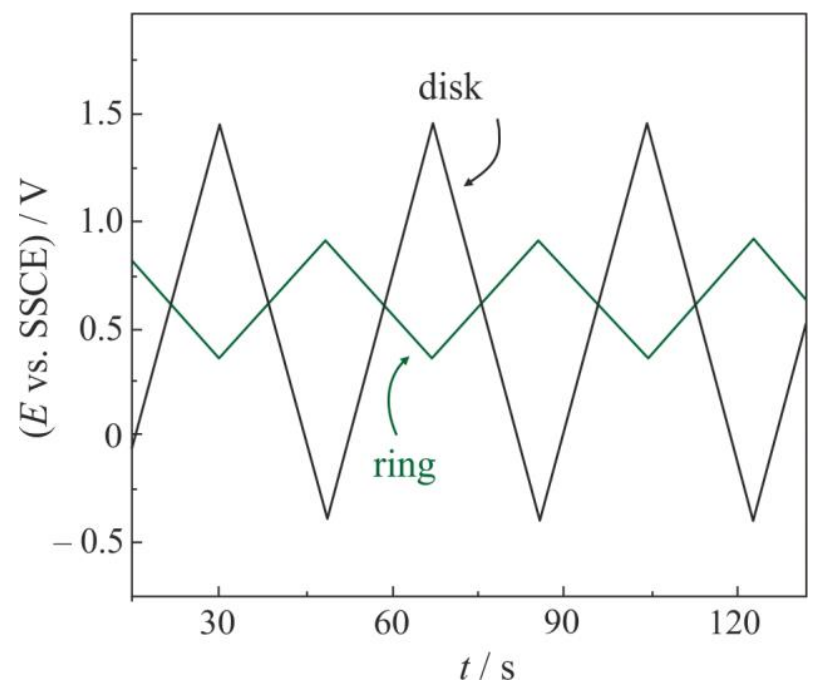

Figure 2. Controlling waveforms of the disk and the ring electrodes. The vertices of the triangular waveform applied to the disk electrode are at -400 and $1450 \mathrm{mV}$, while the ring electrode is polarized in the interval of 910 and $355 \mathrm{mV}$. Sweep rates: $v_{\text {disk }}=100 \mathrm{mV} / \mathrm{s}, v_{\text {ring }}=30 \mathrm{mV} / \mathrm{s}$.

The measurements were carried out by using a gold disk/gold ring RRDE tip, and cyclic voltammograms (CVs) were recorded simultaneously at both working electrodes (disk and ring). The disk electrode was polarized between $-400 \mathrm{mV}$ and $1450 \mathrm{mV}$ (vs. SSCE), while the resulting products were collected by polarizing the ring electrode between $355 \mathrm{mV}$ and $910 \mathrm{mV}$ (vs. SSCE). The frequency (period) of both triangular 
controlling waveforms (i.e., that controlling the disk and that controlling the ring potential) was the same, however the two waveforms were of opposite phase. Thus the perturbing triangular potential waveforms were perfectly synchronized ("synchronous dual cyclic voltammetry" (SDCV), see Fig. 2).

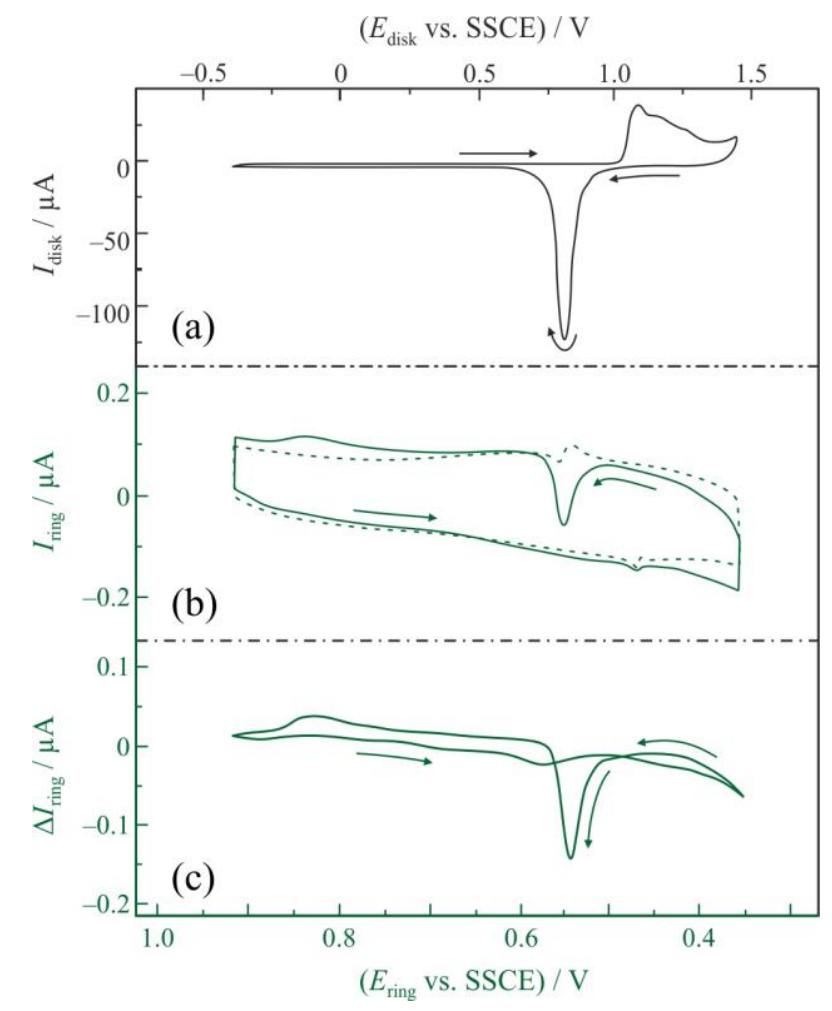

Figure 3. Cyclic voltammograms obtained simultaneously from the disk (a) and ring (b) electrodes. Solid and dashed lines mark the measured CVs when the electrode is rotating (at $\left.500 \mathrm{~min}^{-1}\right)$ and when it does not rotate $\left(0 \mathrm{~min}^{-1}\right)$. The plot in (c) shows the difference between the solid and dashed curves in (b). (Prior to processing, the data were smoothed using an 11 point Savitzky-Golay smoothing algorithm.) $E_{\text {disk }}$ and $E_{\text {ring }}$ are electrode potentials of the ring and the disk, $I_{\text {disk }}$ and $I_{\text {ring }}$ are the corresponding disk and ring currents, $\Delta I_{\text {ring }}$ is the corrected ring current, respectively. Sweep rates: $v_{\text {disk }}=100 \mathrm{mV} / \mathrm{s}$, $v_{\text {ring }}=30 \mathrm{mV} / \mathrm{s}$.

Performing the measurements with rotating $\left(500 \mathrm{~min}^{-1}\right)$ and stationary $\left(0 \mathrm{~min}^{-1}\right)$ RRDE tips, the resulting ring voltammograms were different (compare the continuous and dashed lines, respectively, in Fig. 3(b)). In the absence of rotation, disk-generated products cannot reach the surface of the ring and thus no actual collection takes place. However, ring signals in this case contain all possible features related to electrical crosstalk $(27,28)$. Ring voltammograms measured on a rotated tip on the other hand show collection-related features as well, which are then superimposed on the previous voltammogram. Information related to actual collection can thus be obtained by subtracting the two ring voltammograms (i.e., calculating the difference of rotated and non-rotated ring CVs). This is shown in Fig. 3(c). The careful application of this method allows us to distinguish between electrical cross-talk and actual collection. Note that mass transport does not limit neither the formation nor the reduction of the oxide layer on gold, disk CVs measured on rotating and stationary electrodes are essentially identical. 
A very important advantage of this technique (compared to constant ring potential experiments) is that by the continuous potential cycling, the surface of the ring electrode is kept clean and no loss of detection sensitivity can be experienced.

The cyclic voltammogram recorded at the gold disk, the collection-related ring signal, and the $\left(1 / R_{\mathrm{c}}\right)$ vs. $E$ curve of gold recorded in $0.05 \mathrm{M} \mathrm{H}_{2} \mathrm{SO}_{4}$ solution at sweep rates of $100 \mathrm{mV} \mathrm{s}^{-1}$ are shown in Fig. 4. The change in the radius of curvature of the cantilever is about $0.005 \mathrm{~m}^{-1}$ in the potential range from $-0.4 \mathrm{~V}$ to $1.45 \mathrm{~V}$ vs. SSCE (Fig. 4(c)). This corresponds to $\sim 1.66 \mathrm{~N} / \mathrm{m}$ interface stress change. As it can be seen in Figs. 4(b) and 4(c) the sudden change in the slope of the interfacial stress $v s$. electrode potential curve recorded during the negative-going scan of the cyclic voltammogram coincides with the detection of a reducible species by dual cyclic voltammetry.

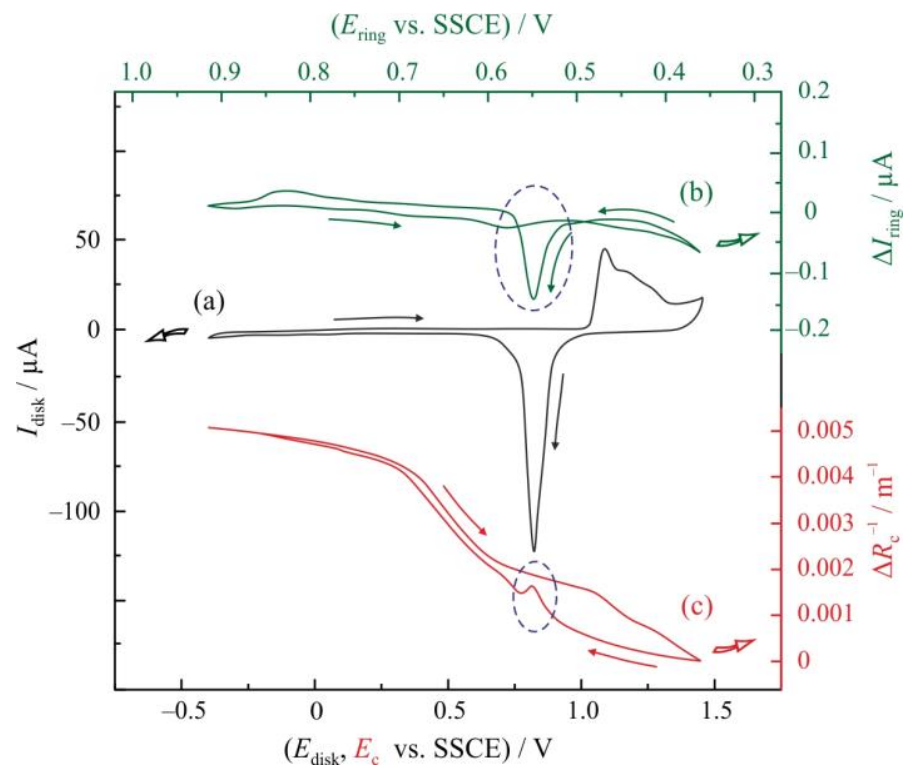

Figure 4. Cyclic voltammogram recorded at the gold disk (a) and the collection-related ring signal (b), calculated as shown in Fig. 3. Electrolyte solution: $0.05 \mathrm{~mol} \cdot \mathrm{dm}^{-3} \mathrm{H}_{2} \mathrm{SO}_{4}$. (c): changes of the curvature $\Delta R_{\mathrm{c}}^{-1}$ of the gold coated cantilever with the electrode potential in the same solution. (The interface stress change $\left(\Delta \gamma_{\mathrm{s}}\right)$ is proportional to $\Delta R_{\mathrm{c}}{ }^{-1}$, the original curve was smoothed using an 11-point Savitzky-Golay filtering algorithm.) $E_{\text {ring, }} E_{\text {disk, }}$, and $E_{\mathrm{c}}$ are electrode potentials of the ring, disk, and the cantilever electrodes, respectively, $I_{\text {disk }}$ is the disk current, $\Delta I_{\text {ring }}$ is the corrected ring current (see Fig. 3(c)). Sweep rates: $v_{\text {disk }}=100 \mathrm{mV} / \mathrm{s}, v_{\text {cantilever }}=100 \mathrm{mV} / \mathrm{s}, v_{\text {ring }}=30 \mathrm{mV} / \mathrm{s}$.

\section{Conclusions}

By the application of dual dynamic voltammetry it could be shown that the sharp change in the slope of the interfacial stress vs. potential curve recorded in the oxide reduction region of gold in sulfuric acid solutions is accompanied by the formation of soluble, electrochemically reducible gold species which can be detected at the ring electrode. Both phenomena (i.e. the formation of a soluble gold species and the sudden change in the slope of the interface stress vs. electrode potential curve) occur within the 
same potential interval suggesting that both may have the same origin or one may be the cause of the other.

\section{Acknowledgments}

This work was supported by the UNKP-17-3 New National Excellence Program of the Ministry of Human Capacities (Hungary), id.: ELTE/12422/82 (2017). Support from the Hungarian Scientific Research Fund - OTKA and the National Research, Development and Innovation Office - NKFI (grant No. K 109036) is gratefully acknowledged.

\section{References}

1. S. Trasatti, R. Parsons, Pure\&Appl. Chem., 58, 437 (1986).

2. K. E. Heusler, G. Láng, Electrochim. Acta, 42, 747 (1997).

3. G. G. Láng and C. A. Barbero, Laser Techniques for the Study of Electrode Processes, F. Scholz, Editor, Springer Verlag, Heidelberg, London, New York (2012).

4. E. M. Gutman, J. Solid State Electrochem., 20, 2929 (2016).

5. G. G. Láng, N. Kovács, S. Vesztergom, M. Ujvári, D. Zalka, K. Szekeres, Tech. Mess., 84, 644 (2017).

6. D. Dickertmann, J. W. Schultze, K. J. Vetter, J. Electroanal. Chem., 55, 429 (1974).

7. H. Angerstein-Kozlowska, B.E. Conway, B. Barnett and J. Moyota, J. Electroanal. Chem., 100, 417 (1979).

8. A. Hamelin, in Modern Aspects of Electrochemistry 16, B.E. Conway, J.O.'M. Bockris and R.E. White, Editors, Ch.1, Plenum Press, New York (1985).

9. M. Sotto, J. Electroanal. Chem., 70, 291 (1976).

10. S. H. Cadle and S. Bruckenstein, Anal. Chem., 46, 16 (1974).

11. S. Vesztergom, M. Ujvári, G. G. Láng, Electrochem. Commun., 13, 378 (2011).

12. B. R. Shrestha, A. Nishikata, T. Tsuru, J. Electroanal. Chem. 665, 33 (2012)

13. S. Cherevko, A. A. Topalov, I. Katsounaros, K. J. J. Mayrhofer, Electrochem. Commun., 28, 44 (2013).

14. S. Cherevko, A. A. Topalov, A. R. Zeradjanin, I. Katsounaros, K. J. J. Mayrhofer, RSC Adv., 3, 16516 (2013).

15. S. Cherevko, A. R. Zeradjanin, A. A. Topalov, G. P. Keeley, K. J. J. Mayrhofer, J. Electrochem. Soc., 161, H501 (2014).

16. S. Cherevko, A. R. Zeradjanin, G. P. Keeley, K. J. J. Mayrhofer, J. Electrochem. Soc., 161, H822 (2014).

17. M. C. Lafouresse, U. Bertocci, C. R. Beauchamp, G. R. Stafford, J. Electrochem. Soc., 159, H816 (2012).

18. G. G. Láng, M. Ujvári, T. A. Rokob, G. Inzelt, Electrochim. Acta, 51, 1680 (2006).

19. S. Vesztergom, N. Kovács, M. Ujvári, G. G. Láng, Tech. Mess., 84, 683 (2017).

20. G. G. Stoney, Proc Roy. Soc. London A32, 172 (1909).

21. G. G. Láng, in Electrochemical Dictionary, A. J. Bard, G. Inzelt, F. Scholz, Editors, p. 59, Springer, Heidelberg, Dordrecht, London, New York (2012). 
22. T. A. Rokob, G. G. Láng, Electrochim. Acta, 51, 93 (2005).

23. G. G. Láng, J. Electrochem. Soc., 163, Y15 (2016).

24. S. Vesztergom, M. Ujvári, G. G. Láng, Electrochem. Commun., 19, 1 (2012).

25. S. Vesztergom, M. Ujvári, G. G. Láng, Electrochim. Acta, 110, 49 (2013).

26. N. Kovács, M. Ujvári, G. G. Láng, P. Broekmann, S. Vesztergom, Instrum. Sci. Tech., 43, 633 (2015).

27. S. Vesztergom, N. Barankai, N. Kovács, M. Ujvári, P. Broekmann, H. Siegenthaler, G. G. Láng, Electrochem. Comm., 68, 54 (2016).

28. S. Vesztergom, N. Barankai, N. Kovács, M. Ujvári, H. Siegenthaler, P. Broekmann, G. G. Láng, J. Solid State Electrochem., 20, 3165 (2016). 